\title{
Polynomial optimization on odd-dimensional spheres
}

\author{
John P. D'Angelo and Mihai Putinar
}

\begin{abstract}
The sphere $S^{2 d-1}$ naturally embeds into the complex affine space $\mathbb{C}^{d}$. We show how the complex variables in $\mathbb{C}^{d}$ simplify the known Striktpositivstellensätze, when the supports are resticted to semi-algebraic subsets of odd dimensional spheres.
\end{abstract}

Mathematics Subject Classification (2000). Primary 14P10; Secondary 32A70.

Keywords. Positive polynomial, Hermitian square, unit sphere, plurisubharmonic function.

\section{Preliminaries}

Let $\mathbb{C}^{d}$ denote complex Euclidean space with Euclidean norm given by $|z|^{2}=$ $\sum_{j=1}^{d}\left|z_{j}\right|^{2}$. The unit, odd dimensional sphere

$$
S^{2 d-1}=\left\{z \in \mathbb{C}^{d} ;|z|=1\right\}
$$

is a particularly important example of a Cauchy-Riemann (usually abbreviated CR) manifold. This note will show how one can study problems of polynomial optimization over semi-algebraic subsets of $S^{2 d-1}$ by using the induced CauchyRiemann structure. Our results can be regarded as multivariate analogues of classical phenomena about positive trigonometric polynomials, known for a long time in dimension one $(d=1)$. They are also related to results concerning proper holomorphic mappings between balls in different dimensional complex Euclidean spaces and the geometry of holomorphic vector bundles.

The second author was supported in part by the Institute for Mathematics and its Applications (Minneapolis) with funds provided by the National Science Foundation. Both authors were partially supported by the National Science Foundation-USA; the first by grant DMS-0500765, the second by grant DMS-0350911. 
A polynomial map $p: \mathbb{C}^{d} \times \mathbb{C}^{d} \rightarrow \mathbb{C}$ is called Hermitian symmetric if

$$
p(z, \bar{w})=\overline{p(w, \bar{z})}
$$

for all $z$ and $w$. By polarization one can recover a Hermitian symmetric polynomial from its real values $p(z, \bar{z})$. We therefore work on the diagonal (where $w=z$ ) and let $\mathcal{H} \subset \mathbb{C}[z, \bar{z}]$ denote the space of Hermitian symmetric polynomials on $\mathbb{C}^{d}$. Note that $\mathcal{H}$ is a real algebra, naturally isomorphic to the polynomial algebra $\mathbb{R}[x, y]$, where $z=x+i y \in \mathbb{R}^{d}+i \mathbb{R}^{d}$. Henceforth we will freely identify a Hermitian symmetric polynomial $P(z, \bar{z})$ with its real form $p(x, y)=P(x+i y, x-i y)$.

We denote by $\Sigma^{2} \mathcal{H}$ the convex cone consisting of sums of squares of Hermitian polynomials. We denote by $\Sigma_{h}^{2} \mathcal{H}$ the convex cone consisting of polynomials which are squared norms of (holomorphic) polynomial mappings. Thus $R \in \Sigma_{h}^{2} \mathcal{H}$ if there exist polynomials $p_{j} \in \mathbb{C}[z]$ such that

$$
R(z, \bar{z})=\sum_{j=1}^{m}\left|p_{j}(z)\right|^{2} .
$$

See [11] and [1] for various characterizations of $\Sigma_{h}^{2} \mathcal{H}$. We have the containment

$$
\Sigma_{h}^{2} \mathcal{H} \subset \Sigma^{2} \mathcal{H}
$$

simply because

$$
|p|^{2}=\left(\frac{p+\bar{p}}{2}\right)^{2}+\left(\frac{p-\bar{p}}{2 i}\right)^{2}=u^{2}+v^{2}
$$

where $u$ and $v$ are the real and imaginary parts of $p$. The containment is strict as illustrated by the following two examples.

Example a). In one variable we define a polynomial $R$ by

$$
R(z, \bar{z})=(z+\bar{z})^{2}=4 x^{2}
$$

It is evidently a square but not in $\Sigma_{h}^{2} \mathcal{H}$. Note that the zero set of an element in $\Sigma_{h}^{2} \mathcal{H}$ must be a complex variety and thus cannot be the imaginary axis.

Example b). In two variables we define $R(z, \bar{z})=\left(\left|z_{1}\right|^{2}-\left|z_{2}\right|^{2}\right)^{2}$. Again $R$ lies in $\Sigma^{2} \mathcal{H}$ but not in $\Sigma_{h}^{2} \mathcal{H}$. Here one can observe that elements of $\Sigma_{h}^{2} \mathcal{H}$ must be plurisubharmonic but that $R$ is not. In 3.3 we will show additionally that $R$ cannot be written as a squared norm on the unit sphere. 
In this paper we are primarily concerned with optimization on the sphere. We therefore first let $I=I\left(S^{2 d-1}\right)$ be the ideal of $\mathcal{H}$ consisting of all polynomials vanishing on $S^{2 d-1}$. We then define

$$
\mathcal{H}\left(S^{2 d-1}\right)=\mathcal{H} / I
$$

and regard it as a space of polynomial functions defined on the sphere. As a matter of fact, each real-valued polynomial $p$ has a representative in $\mathcal{H}\left(S^{2 d-1}\right)$, when $p$ is regarded as a function on the sphere.

In analogy with the above notations we denote by $\Sigma^{2} \mathcal{H}\left(S^{2 d-1}\right)$ the convex cone consisting of sums of squares of Hermitian polynomials on the sphere. We denote by $\Sigma_{h}^{2} \mathcal{H}\left(S^{2 d-1}\right)$ the convex hull of Hermitian squares:

$$
\Sigma_{h}^{2} \mathcal{H}\left(S^{2 d-1}\right)=\operatorname{co}\left\{|p(z)|^{2} ; p \in \mathbb{C}[z]\right\} \quad \bmod I .
$$

Let us pause for a moment and recall a classical one-dimensional result which is guiding our investigation. We include its elementary proof for convenience.

Lemma 1.1 (Riesz-Fejér). A non-negative trigonometric polynomial is the squared modulus of a trigonometric polynomial.

Proof. Let $p\left(e^{i \theta}\right)=\sum_{-d}^{d} c_{j} e^{i j \theta}$ and assume that $p\left(e^{i \theta}\right) \geq 0, \quad \theta \in[0,2 \pi]$. Since $p$ is real-valued $c_{-j}=\overline{c_{j}}$ for all $j$. We set $z=|z| e^{i \theta}$ and extend $p$ to the rational function defined by $p(z)=\sum_{-d}^{d} c_{j} z^{j}$. It follows that $p(z)=\overline{p(1 / \bar{z})}$; furthermore its zeros and poles are symmetrical (in the sense of Schwarz) with respect to the unit circle.

Write $z^{d} p(z)=q(z)$. Then $q$ is a polynomial of degree $2 d$ whose modulus $|q|$ satisfies $|q|=|p|=p$ on the unit circle. In view of the mentioned symmetry one finds

$$
q(z)=c z^{\nu} \prod_{j}\left(z-\lambda_{j}\right)^{2} \prod_{k}\left(z-\mu_{k}\right)\left(z-1 / \overline{\mu_{k}}\right),
$$

where $c$ is a constant, $\left|\lambda_{j}\right|=1$ and $0<\left|\mu_{k}\right|<1$.

Evaluating on the circle and using $\left|\zeta^{2}\right|=|\zeta|^{2}$ we obtain

$$
\begin{gathered}
p\left(e^{i \theta}\right)=\left|p\left(e^{i \theta}\right)\right|=\mid q\left(e^{i \theta} \mid=\right. \\
|c| \prod_{j}\left|e^{i \theta}-\lambda_{j}\right|^{2} \prod_{k} \frac{\left|e^{i \theta}-\mu_{k}\right|^{2}}{\left|\mu_{k}\right|^{2}},
\end{gathered}
$$

and hence $p$ is the squared modulus of a trigonometric polynomial. 
This fundamental lemma has deeply influenced twentieth century functional analysis. For instance the Riesz-Fejér Lemma is equivalent to the spectral theorem for unitary operators; see [28].

When invoking duality, the above is not less interesting. It was in this form that Riesz-Fejér Lemma was first generalized to an arbitrary dimension.

Lemma 1.2. Let $L \in \mathcal{H}\left(S^{2 d-1}\right)^{\prime}$ be a linear functional which is non-negative on $\Sigma_{h}^{2} \mathcal{H}\left(S^{2 d-1}\right)$. Then $L$ is represented by a positive Borel measure supported on the sphere.

The proof has implicitly appeared in the works of Ito [16], Yoshino [31], Lubin [21] and Athavale [3], all dealing with subnormality criteria for commuting tuples of bounded linear operators. Without aiming at completeness, here is the main idea.

Proof. (Sketch) Let $L$ be a non-negative functional on $\Sigma_{h}^{2} \mathcal{H}\left(S^{2 d-1}\right)$. Fix a polynomial $p \in \mathbb{C}[z]$ and consider the functional

$$
f\left(r_{1}^{2}, \ldots, r_{d}^{2}\right) \mapsto L\left(f|p(z)|^{2}\right), \quad f \in \mathbb{R}\left[r_{1}^{2}, \ldots, r_{d}^{2}\right]
$$

where $r_{j}^{2}=\left|z_{j}\right|^{2}$. Since

$$
\begin{gathered}
1-\left|z_{j}\right|^{2}=\sum_{k \neq j}\left|z_{k}\right|^{2} \\
L\left(\prod_{j}\left[\left(1-r_{j}^{2}\right)^{n_{j}} r_{j}^{2 m_{j}}\right]|p|^{2}\right) \geq 0, \quad n_{j}, m_{j} \geq 0 .
\end{gathered}
$$

By a classical result of Haviland, see for instance [2], there exists a positive Borel measure $\mu_{|p|^{2}}$ on the simplex $\Delta$ defined by

$$
\Delta=\left\{\left(r_{1}^{2}, \ldots, r_{d}^{2}\right) ; r_{1}^{2}+\ldots+r_{d}^{2}=1\right\}
$$

with the property

$$
L\left(f|p(z)|^{2}\right)=\int_{\Delta} f d \mu_{|p|^{2}} .
$$

The total mass of $\mu_{|p|^{2}}$ is $L\left(|p|^{2}\right)$.

By polarization, one can define complex valued measures by

$$
L(f p \bar{q})=\int_{\Delta} f d \mu_{p \bar{q}}, \quad f \in \mathbb{R}\left[r_{1}^{2}, \ldots, r_{d}^{2}\right], p, q \in \mathbb{C}[z],
$$

so that the sesqui-linear $\operatorname{kernel}(p, q) \mapsto \mu_{p \bar{q}}$ is positive semi-definite. 
In short, the functional $L$ can be extended to the linear space of functions (on the sphere) of the form

$$
F(r, z)=\sum_{|\alpha| \leq n} c_{\alpha}(r) z^{\alpha},
$$

where $c_{\alpha}(r)$ are bounded, Borel measurable functions on the simplex $\Delta$. The extended functional $\tilde{L}$ still satisfies

$$
\tilde{L}\left(|F(r, z)|^{2}\right) \geq 0 .
$$

Next we pass to polar coordinates $z_{j}=r_{j} \omega_{j},\left|\omega_{j}\right|=1$ and remark that multiplication by $\omega_{j}$ satisfies the isometric condition

$$
\tilde{L}\left(\left|\omega_{j} F(r, z)\right|^{2}\right)=\tilde{L}\left(|F(r, z)|^{2}\right) .
$$

Thus, we can further extend the functional $\tilde{L}$ to all polynomials in $r$ and $\omega, \bar{\omega}$, so that

$$
\tilde{L}\left(\left|\omega_{j}^{-1} F(r, z)\right|^{2}\right)=\tilde{L}\left(|F(r, z)|^{2}\right)
$$

and

$$
\tilde{L}\left(|p(r, \omega, \bar{\omega})|^{2}\right) \geq 0 .
$$

We refer to [31] or [29] for the details how this extension is constructed. By rewriting the latter positivity condition we have in particular

$$
\tilde{L}\left(|h(z, \bar{z})|^{2}\right) \geq 0, \quad h \in \mathbb{C}[z, \bar{z}],
$$

whence, by the Stone-Weierstrass Theorem and the Riesz Representation Theorem, the functional $\tilde{L}$ is represented by a positive Borel measure, supported on the sphere.

The representing measure is unique by the Stone-Weierstrass Theorem.

\section{A Striktpositivstellensatz}

We now turn to the basic question considered in this paper. We are given a finite set of real polynomials in $2 d$ variables $p, q_{1}, \ldots, q_{r}$, or equivalently, Hermitian symmetric polynomials in $d$ complex variables. We suppose that $p(z, \bar{z})$ is strictly positive on the subset of $S^{2 d-1}$ where each $q_{j}$ is nonnegative. Can we write $p$ as a weighted sum of squared norms with $q_{i}$ as weights, as the real affine Striktpositivstellensatz (see for instance [22]) suggests? The answer is yes, and we can offer at least two different reasons why it is so. 
Theorem 2.1. Let $p, q_{1}, \ldots, q_{r} \in \mathbb{R}[x, y]$, where $x+i y=z \in \mathbb{C}^{d}$. If

$$
\left(|z|=1, q_{i}(z, \bar{z}) \geq 0,1 \leq i \leq r\right) \Rightarrow(p(z, \bar{z})>0)
$$

then

$$
p \in \Sigma_{h}^{2}+q_{1} \Sigma_{h}^{2}+\ldots+q_{r} \Sigma_{h}^{2}+I\left(S^{2 d-1}\right) .
$$

First we discuss the history of such Hermitian squares decompositions, in the case where there are no constraints. A Hermitian symmetric polynomial $p$ is called bihomogeneous of degree $(m, m)$ if

$$
p(\lambda z, \overline{\lambda z})=|\lambda|^{2 m} p(z, \bar{z})
$$

for all complex numbers $\lambda$ and all $z \in \mathbb{C}^{d}$. The values of a bihomogeneous polynomial are determined by its values on the sphere. When $p$ is bihomogeneous and strictly positive on the sphere, Quillen [27] proved that there is an integer $k$ and a homogeneous polynomial vector-valued mapping $h(z)$ such that

$$
|z|^{2 k} p(z, \bar{z})=|h(z)|^{2} \text {. }
$$

This result was discovered independently by the first author and Catlin [6] in conjunction with the first author's work on proper mappings between balls in different dimensions. The proof in [6] uses the Bergman projection and some facts about compact operators, and it generalizes to provide an isometric imbedding theorem for certain holomorphic vector bundles [7].

It is worth noting that the integer $k$ and the number of components of $h$ can be arbitrarily large, even for polynomials $p$ of total degree four in two variables. The result naturally fits into the phenomena encoded into the old or recent Positivestellensätze, see for instance [22]. For the specific case of Hermitian polynomials on spheres see [8] for considerable discussion and generalizations.

Using a process of bihomogenization, Catlin and the first author (see [6], [8] and [9]) proved that if $p$ is arbitrary (not necessarily bihomogeneous) and strictly positive on the sphere, then $p$ agrees with a squared norm on the sphere; in other words, $p \in \Sigma_{h}^{2}+I\left(S^{2 d-1}\right)$. Thus Theorem 1 holds when there are no constraints. Our proof of Theorem 1 first considers the case of no constraints, but we approach this case in a completely different manner.

Strict positivity is required for these results. The polynomial $\left(\left|z_{1}\right|^{2}-\left|z_{2}\right|^{2}\right)^{2}$ is bihomogeneous and nonnegative everywhere, but there is no element in $\Sigma_{h}^{2}$ agreeing with it on the sphere. See Example 3.3 below. 
Proof. (of Theorem 1) Suppose first that no $q_{i}$ 's are present and assume by contradiction that $p \notin \Sigma_{h}^{2}$, all regarded as elements of $\mathcal{H}\left(S^{2 d-1}\right)$. Since the constant function 1 belongs to the algebraic interior of the convex cone $\mathcal{H}\left(S^{2 d-1}\right)$, the separation lemma due to Eidelheit-Kakutani $[12,17]$ provides a linear functional $L \in \mathcal{H}\left(S^{2 d-1}\right)^{\prime}$, satisfying both $L(1)>0$ and

$$
L(p) \leq 0 \leq\left. L\right|_{\Sigma_{h}^{2}}
$$

According to Lemma 2, there exists a positive Borel measure $\mu$, supported on the unit sphere, which represents $L$. Therefore

$$
0 \geq L(p)=\int p d \mu>0,
$$

a contradiction.

The proof of the general case is similar, with the difference that we have to prove that the support of the measure $\mu$ is contained in the non-negativity set defined by the functions $q_{i}$. To this aim, fix an index $i$, and remark that

$$
\int q_{i}|p|^{2} d \mu \geq 0
$$

for all $p \in \mathbb{C}[z]$. Now, by the first case, every positive polynomial $P(z, \bar{z})$ is in the convex hull of the Hermitian squares, whence

$$
\int q_{i} P(z, \bar{z}) d \mu \geq 0
$$

whenever $P(z, \bar{z})>0$ on the sphere, that is whenever $P(z, \bar{z}) \geq 0$ on the sphere. In view of Stone-Weierstrass Theorem, every continuous functions $f$ on the sphere can be uniformly approximated by real polynomials. In particular, we infer

$$
\int q_{i} f^{2} d \mu \geq 0, \quad f \in C\left(S^{2 d-1}\right) .
$$

But this inequality holds only if the support of $\mu$ is contained in the non-negativity set $q_{i}(z, \bar{z}) \geq 0$.

\section{Examples}

\subsection{Optimization on the closed disk}

The following simple example shows that Hermitian sums of squares do not suffice as positivity certificates on more general semi-algebraic sets. Specifically, let

$$
P(z, \bar{z})=1-\frac{4}{3}|z|^{2}+a|z|^{4}
$$


with $\frac{1}{3}<a$. Note that

$$
P(z, \bar{z})=\left(1-\frac{2}{3}|z|^{2}\right)^{2}+\left(a-\frac{4}{9}\right)|z|^{4}
$$

and hence $P \in \Sigma^{2} \mathcal{H}$ when $a \geq \frac{4}{9}$. Hence we assume $\frac{1}{3}<a<\frac{4}{9}$. The polynomial $1-\frac{4}{3} t+a t^{2}$ is decreasing for $0<t<1$ when $a<\frac{2}{3}$; therefore $|z| \leq 1$ implies $P(z, \bar{z}) \geq 1+a-\frac{4}{3}>0$.

On the other hand,

$$
P \notin \Sigma_{h}^{2}+\left(1-|z|^{2}\right) \Sigma_{h}^{2} .
$$

To see that $P$ is not in this set, we apply the hereditary calculus. See [1] for details. We replace $z$ with a contractive operator $T$ and replace $\bar{z}$ with $T^{*}$. We follow the usual convention of putting all $T^{*}$ 's to the left of the powers of $T$. If $P$ were in this set, we would obtain

$$
\|T\| \leq 1 \Rightarrow p(T, \bar{T}) \geq 0 .
$$

In particular let $T$ be the $2 \times 2$ Jordan block with 1 above the diagonal. We obtain a contradiction by computing that $P\left(T, T^{*}\right)$ is the diagonal matrix with eigenvalues 1 and $-\frac{1}{3}$.

On the other hand, the larger convex cone $\Sigma^{2}+\left(1-|z|^{2}\right) \Sigma_{h}^{2}$ is appropriate in this case, see [24, 26].

\subsection{Squared norms}

Recall that $\Sigma_{h}^{2} \mathcal{H}$ denotes the convex cone consisting of polynomials which are squared norms of (holomorphic) polynomial mappings. In all dimensions the zero set of an element in $\Sigma_{h}^{2} \mathcal{H}$ must be a complex variety.

Suppose $R(z, \bar{z}) \geq 0$ for all $z$. Even in one dimension we cannot conclude that $R \in \Sigma_{h}^{2} \mathcal{H}$. We noted earlier, where $x=\operatorname{Re}(z)$, the example

$$
R(z, \bar{z})=(z+\bar{z})^{2}=4 x^{2} .
$$

The zero set of $R$ is the imaginary axis, which has no complex structure. In one dimension of course, the zero set of an element in $\Sigma_{h}^{2} \mathcal{H}$ must be either all of $\mathbb{C}$ or a finite set.

Things are more complicated and interesting in higher dimensions. Consider the following example from [8]. Define a Hermitian bihomogeneous polynomial in three variables by

$$
p(z, \bar{z})=\left(\left|z_{1} z_{2}\right|^{2}-\left|z_{3}\right|^{4}\right)^{2}+\left|z_{1}\right|^{8} .
$$


This polynomial $p$ is nonnegative for all $z$, and its zero set is the complex plane given by $z_{1}=z_{3}=0$ with $z_{2}$ arbitrary. Yet $p$ is not a sum of squared moduli; even more striking is that $p$ cannot be written as the quotient $\frac{|a(z)|^{2}}{|b(z)|^{2}}$ where $a$ and $b$ are sums of squared moduli. See [10] for additional information on this example and several tests for deciding whether a non-negative polynomial $R$ can be written as a quotient of squared norms. See [30] for a necessary and sufficient condition involving the zeroes of $R$.

We give an additional example in one dimension. Define $p$ by

$$
p(z, \bar{z})=1+b z^{2}+\bar{b} \bar{z}^{2}+c|z|^{2}+|z|^{4} .
$$

The condition for being a quotient of squared norms is that one of the following three statements holds:

$$
\begin{aligned}
& c>2|b|^{2}-2, \\
& b=0, c>-2, \\
& |b|=1, c=0 .
\end{aligned}
$$

The condition for being nonnegative is simpler: $c \geq 2|b|-2$.

\subsection{Proof of Example b).}

We claimed earlier that the polynomial $\left(\left|z_{1}\right|^{2}-\left|z_{2}\right|^{2}\right)^{2}$ is bihomogeneous and nonnegative everywhere, but there is no element in $\Sigma_{h}^{2}$ agreeing with it on the sphere.

Proof. Put $R(z, \bar{z})=\left(\left|z_{1}\right|^{2}-\left|z_{2}\right|^{2}\right)^{2}$, and let $V(R)$ denote its zero set. We note that $V(R) \cap S^{2 n-1}$ is the torus $T$ defined by $\left|z_{1}\right|^{2}=\left|z_{2}\right|^{2}=\frac{1}{2}$. Suppose for some polynomial mapping $z \rightarrow P(z)$ we have $R=|P|^{2}$ on the unit sphere. Note first that the zero set of $|P|^{2}$ is a complex variety. We have $|P(z)|^{2}=0$ for $z \in T$. We claim that $P$ is identically zero. For each fixed $z_{2}$ with $\left|z_{2}\right|=1$, the vector-valued polynomial mapping $z_{1} \rightarrow P\left(z_{1}, z_{2}\right)$ vanishes on the circle $\left|z_{1}\right|^{2}=\frac{1}{2}$ and hence vanishes identically. Since $z_{2}$ was an arbitrary point with $\left|z_{2}\right|^{2}=\frac{1}{2}$ we conclude that the mapping $\left(z_{1}, z_{2}\right) \rightarrow P\left(z_{1}, z_{2}\right)$ vanishes whenever $z_{1} \in \mathbf{C}$ and $z_{2}$ lies on a circle. By symmetry it also vanishes with the roles of the variables switched. It follows that the zero set of $P$ (which is a complex variety) is at least three real dimensions, and hence $P$ vanishes identically. Since $R$ does not vanish identically on the sphere we obtain a contradiction. 


\subsection{Example}

There exist non-negative polynomials $R$ such that $R$ is not in $\Sigma_{h}^{2} \mathcal{H}$, yet there is a positive integer $N$ for which $R^{N} \in \Sigma_{h}^{2} \mathcal{H}$. The bihomogeneous polynomial $R_{\lambda}$ given by

$$
R_{\lambda}(z, \bar{z})=\left(\left|z_{1}\right|^{2}+\left|z_{2}\right|^{2}\right)^{4}-\lambda\left|z_{1} z_{2}\right|^{4}
$$

satisfies this property whenever $\lambda<8$. See [30]. For $\lambda<16, R_{\lambda}>0$ on the sphere. By Theorem 1 it agrees with a squared norm on the sphere.

\section{References}

[1] J. AGLER, J.E. McCARTHY, Pick interpolation and Hilbert function spaces. Graduate Studies in Mathematics. 44. Providence, RI: American Mathematical Society (2002).

[2] N. I. AKHIEZER, The Classical Moment Problem. Oliver and Boyd, Edinburgh and London (1965).

[3] A. ATHAVALE, Holomorphic kernels and commuting operators. Trans. Amer. Math. Soc. 304, 101-110(1987).

[4] J.A., BALL, T.T. TRENT, Unitary colligations, reproducing kernel Hilbert spaces, and Nevanlinna-Pick interpolation in several variables. J. Funct. Anal. 157, No.1, 1-61 (1998).

[5] A. BARVINOK, Integration and optimization of multivariate polynomials by restriction onto a random subspace. Foundations of Computational Mathematics, to appear.

[6] D.W. CATLIN, J.P. D'ANGELO, A stabilization theorem for Hermitian forms and applications to holomorphic mappings, Math. Res. Lett. 3, No. 2, 149-166 (1995).

[7] D.W. CATLIN, J.P. D'ANGELO, An isometric imbedding theorem for holomorphic bundles. Math. Res. Lett. 6, No.1, 43-60 (1999).

[8] J.P. D'ANGELO, Inequalities from Complex Analysis, Carus Mathematical Monographs, No. 28, Mathematical Assn. of America (2002).

[9] J.P. D'ANGELO, Proper holomorphic mappings, positivity conditions, and isometric imbedding J. Korean Math Soc. 40, No. 3, 341-371 (2003).

[10] J.P. D'ANGELO, Complex variables analogues of Hilbert's seventeenth problem, International Journal of Mathematics 16, 609-627 (2005).

[11] J.P. D'ANGELO and D. VAROLIN, Positivity conditions for Hermitian symmetric functions, Asian J. Math 8, 215-232 (2004).

[12] M. EIDELHEIT, Zur Theorie der konvexen Mengen in linearen normierten Räumen. Studia Math. 6, 104-111 (1936). 
[13] J. ESCHMEIER, M. PUTINAR, Spherical contractions and interpolation problems on the unit ball. J. Reine Angew. Math. 542, 219-236 (2002).

[14] C. FOIAS, A.E. FRAZHO, The commutant lifting approach to interpolation problems. Operator Theory: Advances and Applications, 44. Basel. Birkhäuser, (1990).

[15] J.W.HELTON, S. McCULLOUGH, M. PUTINAR, Non-negative hereditary polynomials in a free *-algebra. Math. Zeitschrift 250, 515-522 (2005)

[16] T. ITO, On the commutative family of subnormal operators, J. Fac. Sci. Hokkaido Univ. 14, 1-15(1958)

[17] S. KAKUTANI, Ein Beweis des Satzes von M. Eidelheit über konvexe Mengen. Proc. Imp. Acad. Tokyo 13, 93-94 (1937).

[18] M.G. KREIN, M.A. NAIMARK, The method of symmetric and Hermitian forms in the theory of separation of the roots of algebraic equations. (Translated from the Russian by O. Boshko and J. L. Howland). Linear Multilinear Algebra 10, 265-308 (1981).

[19] J.E.McCARTHY, M. PUTINAR, Positivity aspects of the Fantappiè transform. J. d'Analyse Math. 97, 57-83(2005)

[20] J.B. LASSERRE, Global optimization with polynomials and the problem of moments. SIAM J. Optim. 11, No.3, 796-817 (2001).

[21] A. LUBIN, Weighted shifts and commuting normal extension, J. Austral. Math. Soc. 27, 17-26(1979)

[22] A. PRESTEL, C.N. DELZELL, Positive polynomials. From Hilbert's 17th problem to real algebra. Springer Monographs in Mathematics. Berlin: Springer, (2001).

[23] A. PRESTEL, Representation of real commutative rings. Expo. Math. 23, 89-98 (2005).

[24] M. PUTINAR, Sur la complexification du probléme des moments. C. R. Acad. Sci., Paris, Série I 314, No.10, 743-745 (1992).

[25] M.PUTINAR, Positive polynomials on compact semi-algebraic sets. Indiana Univ. Math. J. 42, No.3, 969-984 (1993).

[26] M. PUTINAR, On Hermitian polynomial optimization. Arch. Math. 87, 41-5(2006)

[27] D.G. QUILLEN, On the representation of Hermitian forms as sums of squares. Invent. Math. 5, 237-242 (1968).

[28] F. RIESZ, B. SZ.-NAGY, Functional analysis. Transl. from the 2nd French ed. by Leo F. Boron. Reprint of the 1955 orig. publ. by Ungar Publ. Co., Dover Books on Advanced Mathematics. New York: Dover Publications, Inc., (1990).

[29] B. SZ.-NAGY, C. FOIAS, Analyse harmonique des opérateurs de l'espace de Hilbert. Budapest: Akademiai Kiado; Paris: Masson et Cie, 1967. 
[30] D. VAROLIN, Geometry of Hermitian algebraic functions: quotients of squared norms. American J. Math, to appear.

[31] T. YOSHINO, On the commuting extensions of nearly normal operators, Tohoku Math. J. 25, 163-172 (1973)

John P. D'Angelo

(J.D.) Department of Mathematics, University of Illinois at Urbana-Champaign, Urbana, IL 61801

e-mail: jpda@math.uiuc.edu

Mihai Putinar

(M.P.) Mathematics Department, University of California, Santa Barbara, CA 93106

e-mail: mputinar@math .ucsb.edu 